\title{
Effects of Non-thermal Electrons from ECCD on ECE Temperature Measurements for ITER
}

\author{
P V Subhash ${ }^{1, a}$, Hitesh Kumar B. Pandya ${ }^{1}$, Ravinder Kumar ${ }^{1}$, and P. Vasu ${ }^{1}$ \\ ITER-India, Institute for Plasma Research, Gandhinagar, Gujarat, INDIA
}

\begin{abstract}
In tokamaks, the radial temperature profile measured using Electron $\mathrm{Cy}$ clotron Emission (ECE) diagnostics are affected by many phenomena like harmonics overlap, relativistic down shifting, presence of non-thermals etc. In this paper we have estimated effects of a small non-thermal electron population on measured temperature profile for ITER-Scenario 2. For ITER like plasma, radial temperature profiles can be obtained from the second harmonic ECE spectrum. It is possible that, higher harmonics produced from the non-thermals can be relativistically downshifted to second harmonics and introduce error in the measured temperature profile. Generally Non-thermals are produced from Electron Cyclotron Resonance heating (ECRH), Electron Cyclotron Current Drive (ECCD) etc. In the present study the non-thermals are assumed to be produced from proposed ECCD, which is being considered for suppressing Neoclassical Tearing Modes (NTM). We have ignored any other source of non-thermals in the present study. All the numerical calculations reported in this paper is performed using NOTEC computer code which is capable of handling non-thermal populations. The locations and spatial extents of non-thermals are taken from previous report on optimization study of the ITER ECRH top launcher. The non-thermals are assumed to be centered around safety points $\mathrm{q}=1, \mathrm{q}=1.5$ and $\mathrm{q}=2$, where the ECCD is expected to be used for suppressing the NTMs. The main results of the present study are summarized below. In the first part of the paper we present the results for temperature measurement with out non-thermal populations for the purpose of validation. Secondly the rage of higher harmonic frequencies (due to nonthermals) which possibly reach antenna and induce error in the temperature measurement are identified and the corresponding energies of non-thermal populations are calculated analytically. This calculations are further checked by simulations using NOTEC code. Finally non-thermal populations are seeded in the plasma with fraction and energies of non-thermals are varied in a parametric form. The parametric range of energies are initially bracketed by the analytical calculations explained above. The resultant temperature profiles and error in the measured temperatures will be presented.
\end{abstract}

\section{Introduction}

Electron Cyclotron Emission (ECE) measurement is considered as one of the reliable technique for measurement of radial profile of electron temperature in tokamaks. A comprehensive review of physical and technical aspects are available from Reference [1]. But for high temperature tokamaks like ITER and JET, the capabilities of this method is limited by various effects like harmonic overlap, polarization scrambling, relativistic frequency downshifting etc [2]. All this effects in combination makes measured temperature profile unreliable to some extent. Thus quantifying the induced errors due to various effects is required for proper interpretation of measured ECE spectrum. This paper attempts to quantify the distortion induced due to relativistic down shifting of higher harmonics. In tokamaks like ITER higher harmonics exist due to the electron cyclotron emission from high energetic

\footnotetext{
a e-mail: getsubhash@gmail.com
} 
non-thermals populations. The non-thermal electrons are directly produced mainly from two sources. Firstly because of the Electron Cyclotron Resonance Heating (ECRH) and secondly due to Electron Cyclotron Current Drive (ECCD). ECCD is proposed to use in ITER for stabilizing Neoclassical Tearing Modes (NTM). Stabilizing NTMs demands localized heating using ECCD at various safety points where NTMs are expected. Further details of proposed ECCD and an optimization study for ECCD launcher location can be obtained from Reference [3]. Here we present the numerical calculations for the error induced in the measured radial temperature profiles due to ECCD which is to be used in ITER. As the energy and fraction of the non-thermals are not available, we have done a parametric study on these two parameters. But the parametric regime of the energy relevant for ITER tokamak is initially calculated analytically.

This paper is organized as follows. In Section 2 a brief discribtion of the problem and details of the numerical method are provided. This section also include some results for the case with out nonthermals. In Section 3, a methodology to restrict the energy range of non-thermals will be presented, which is then used for obtaining a parametric space. Finally, the results of the parametric study will be presented in Section 4.

\section{Numerical model and problem discribtion}

All the simulation results presented in this paper is carried out using NOTEC computer code. It is basically a 3D code which calculate the measured spectra using antenna pattern, reflection, refraction and non-thermal populations[4]. A combination of ray tracing and transport solver is used in the code. The procedure of calculation is described below. Using a known antenna pattern several rays, which describe the particular pattern are launched. The transport co-efficient are calculated along all the rays. This is then used for calculating emission and absorption intensity for various locations. This process will be repeated for many frequencies to obtain the spectrum. The derived spectrum is then used for calculating radial temperature profile with an assumption $T_{\text {rad }} \sim T_{\text {measured }}$. Further details about the code can be obtained from Ref [4].

The numerical calculations are performed with and with out non-thermal electrons. For all the cases base profiles for density and temperatures are taken as that of ITER scenario-2. Initial temperature and density profiles are shown in figures 12.

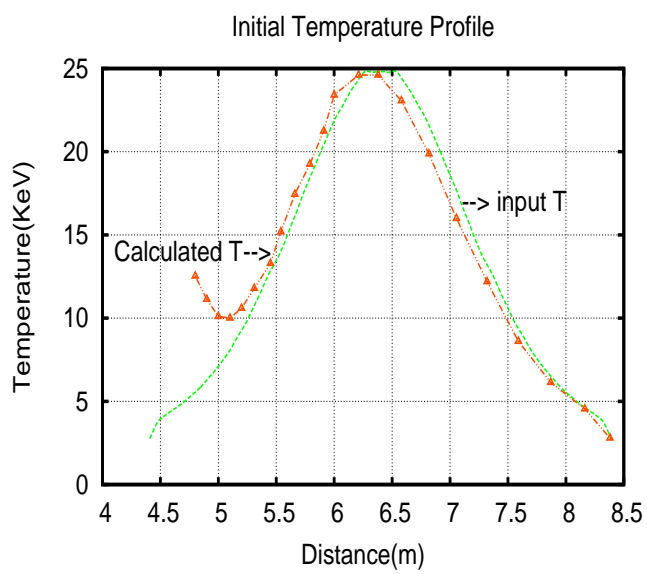

Fig. 1. Temperature Profile, It should be noted that green curve denote input temperature profile and red is calculated with NOTEC 


\section{EC-17 Workshop}

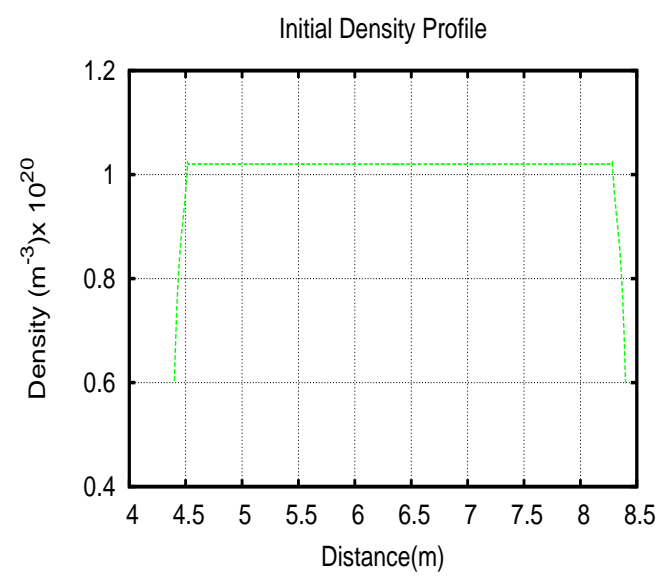

Fig. 2. Radial density profile

The calculated temperature is also shown in figure 1. It should be noted that the calculated temperature and base (input) temperature are slightly different at high field side. This is because of mode overlapping effect.

For the case of ITER radial temperature profiles are calculated from first harmonics Ordinary Mode $(\mathrm{OM})$ and second harmonics Extra-Ordinary Mode (XM). In the present work we used first harmonics ordinary mode for the radial temperature measurement. The non-thermal populations produced due to ECCD can emit radiation with frequency in the higher harmonics range. In the plasma this higher harmonics can be relatively down shifted to first harmonics OM modes. Further this radiations can be collected by the antenna and induce error in the measured temperature.

In the parametric study reported in Section 3, the base profiles are seeded with non-thermal electrons at location with safety factor $q=1$. The energy of the non-thermals and fraction of the nonthermals are varied.

\section{Selection of Parametric Regime}

For the calculations of effect of non-thermal electrons on measured temperature one required to supply energy of the non-thermals and fraction of the non-thermals along with the locations of non-thermals. Ideally this can be obtained by detailed ECCD transport calculations which is beyond the scope of this paper. Instead of doing so we have performed a parametric study by varying energy and fractions of the non-thermals. The fractions of the non-thermal populations are varied from $1 \%$ to $3 \%$. While the parametric regime for the energy of the non-thermals are initially bracketed analytically as described below.

The location of non-thermals are fixed around radial location of the plasma with safety factor $\mathrm{q}=1$. This radial location corresponds to normalized flux co-ordinate 0.43 .

One need not to consider the all the relativistically downshifted radiations, as most of them will not reach to the antenna. A restriction on frequency range can be obtained as follows. All the frequencies below the the natural thermal re-absorption frequency will be absorbed by the plasma. Hence this will provide a limit on frequency below this, radiations will not reach to the antenna. Similarly an upper bound is also exist at upper cut-off frequency. For the case of emission perpendicular to the magnetic field frequency of emission is given by [5]

$$
\omega=n \frac{e B_{0} R_{0}}{m_{e}} \frac{1}{R \gamma}
$$


Here $B_{0}$ and $R_{0}$ are field and major radius at plasma axis while 'n' is the harmonic number. In the present case $\mathrm{n}$ is 2 , as we are interested in down shifting of second harmonics. It should be noted that amount of down shifting will be determined by relativistic parameter $\gamma$. Considering electron rest energy of $511 \mathrm{KeV}$ the above equation can be re written to obtain the radius of emission as [5].

$$
R=n \frac{\omega_{0}}{\omega} R_{0} \frac{511}{511+K}
$$

with $K=m_{e} c^{2}(\gamma-1)$.

The above two equation together is used for an initial bracketing of the energy for the parametric study as shown in Figure 3. From the figure 3 it can be noted that energy of the non-thermals with in the range of $100 \mathrm{KeV}$ to $225 \mathrm{KeV}$ is only important for the present study.

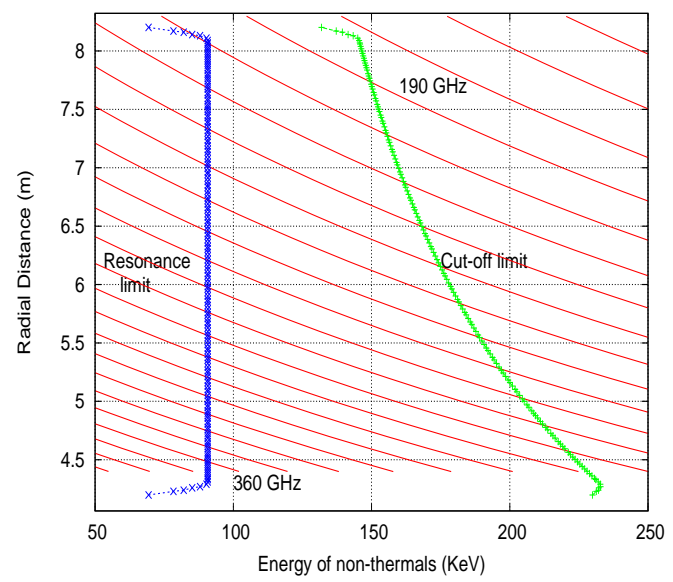

Fig. 3. The emission location in the plasma (for various frequencies) is plotted as the function of energy of the non-thermals. Also lower and upper bound on frequency corresponds to thermal re absorption and upper cut-off is shown

\section{Error induced in the measured temperature because of the non-thermals}

In this section detailed results of the parametric study will be presented. As explained in the last section fraction of the non-thermals are varied form $1 \%$ to $3 \%$ with a step of $1 \%$. While energy is varied from $100 \mathrm{KeV}$ to $225 \mathrm{KeV}$ with a step of $25 \mathrm{KeV}$.

Figures 4 to 6 shows the measured radial temperature profiles for different fractions. Each plot is the radial temperature profiles for one fraction. Different curves corresponds to different energy of non-thermals.

From the figures 4 to 6 followings points are note worthy. All the measured temperature profiles around the central regime and in the high field sides are severely distorted. For the case with fraction of non-thermal at 3\% the measured temperature in the high field side is around $100 \%$ to $300 \%$ is higher than base temperature. While in the central regime it is around $70 \%$. For a fixed value of fraction of non-thermals the error increases with energy of non-thermals. But it should also be noted that the difference between error decrees as we go towards higher energies. For example the difference between error for the $200 \mathrm{KeV}$ and $225 \mathrm{KeV}$ is much lesser than that between $100 \mathrm{KeV}$ and $125 \mathrm{KeV}$. Finally as the fraction of the non-thermals increases as expected error also increases monotonically. For all the cases, in the low field regime some radial locations are still not affected. 


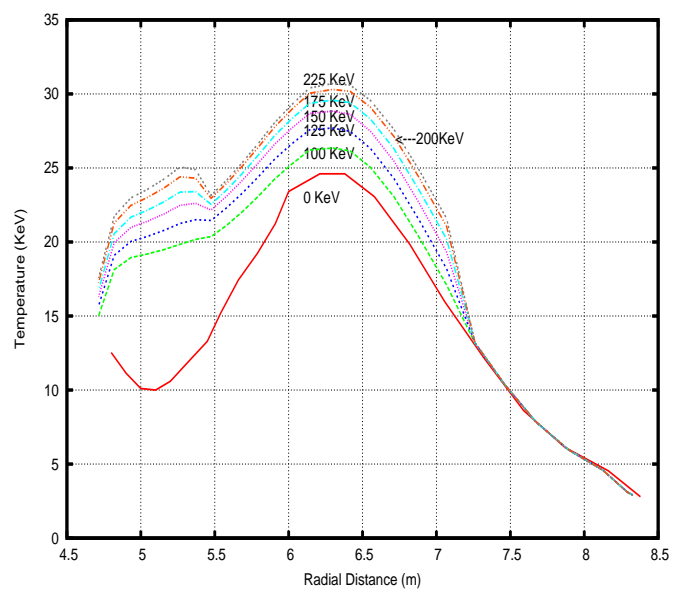

Fig. 4. Radial temperature profiles with non-thermals for different energy for $1 \%$ of local non-thermal density

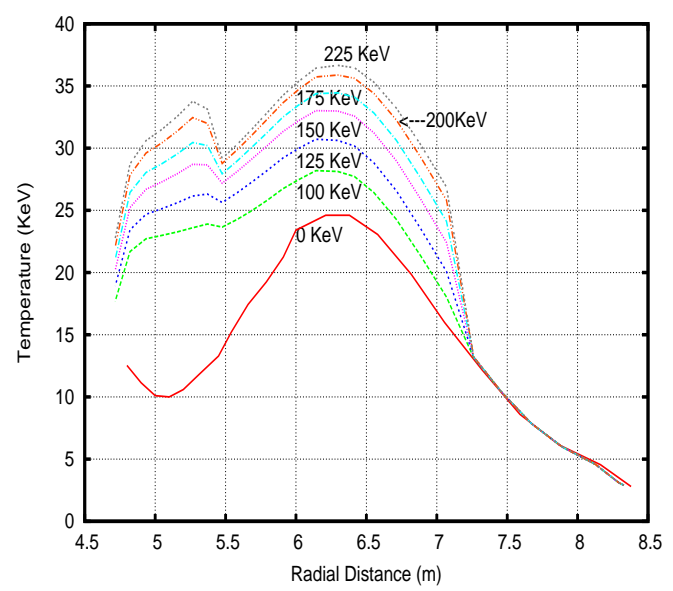

Fig. 5. Radial temperature profiles with non-thermals for different energy for $2 \%$ of local non-thermal density

\section{Conclusion}

The effect of localized non-thermals possibly produced due to local ECCD heating for stabilizing NTMs are numerically treated. This is performed in a parametric study for energy and fraction of non-thermals. The initial bracketing of energy of non-thermals are analytically done. The relativistic down shifting of radiation emitted by non-thermals are found to induce high error in the measured radial temperature profiles. The profiles in the central as well as high field side is severely distorted with $70 \%$ to $300 \%$ error. While some radial location in the low field side towards the edge remains unaffected. As expected increasing the fraction of non-thermals results higher error. The difference in error between different energy of non-thermals are found to decrease as we move towards higher energies.

\section{Acknowledgment}

We acknowledge the use of NOTEC computer code under a license agreement with FOM -Institute voor Plasma Fysica. 


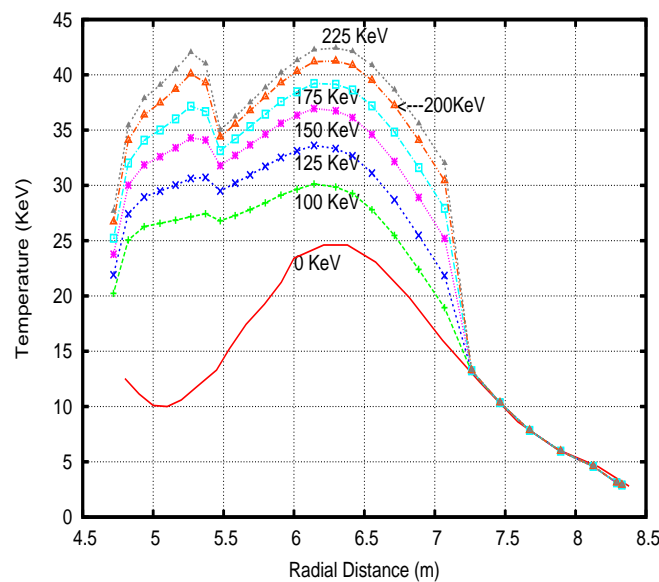

Fig. 6. Radial temperature profiles with non-thermals for different energy for 3\% of local non-thermal density

\section{References}

1. M. Bornatici, et al, Nucl. Fusion 23,(1983), 1153.

2. D. V. Bartlett et al, Physics Issues of ECE and ECA for ITER JET (46) (1995) P-95

3. G. Ramponi et.al. Optimization of the ITER ECRH Top Launcher, IAEA Techn. Meeting on. ECRH Physics and Technology for ITER, (2006).

4. R. M. J. Sillen et.al, manual of 'NOTEC, a Code to Simulate Electron Cyclotron Emission Spectra of Plasma Which Include Non-thermal Populations, FOM Instituut Voor Plasmafysica (1987)

5. M. Brusati et.al. Nucl. Fusion 341 (1994),23 